\title{
BMJ Open Experiences and perceived health benefits of individuals with a disability participating in sport: A systematic review protocol
}

\author{
Beth Aitchison, ${ }^{1}$ Alison Rushton (D) , ${ }^{1,2}$ Paul Martin, ${ }^{3}$ Andrew Soundy (D) , ${ }^{1}$ \\ Nicola R Heneghan (D) ${ }^{1}$
}

To cite: Aitchison $B$,

Rushton A, Martin P, et al. Experiences and perceived health benefits of individuals with a disability participating in sport: A systematic review protocol. BMJ Open 2020;10:e038214. doi:10.1136/ bmjopen-2020-038214

- Prepublication history and additional material for this paper is available online. To view these files, please visit the journal online (http://dx.doi.org/10. 1136/bmjopen-2020-038214).

Received 02 March 2020 Revised 13 August 2020 Accepted 21 October 2020

Check for updates

(c) Author(s) (or their employer(s)) 2020. Re-use permitted under CC BY-NC. No commercial re-use. See rights and permissions. Published by BMJ.

${ }^{1}$ Centre of Precision Rehabilitation for Spinal Pain (CPR Spine), School of Sport, Exercise and Rehabilitation Sciences, College of Life and Environmental Sciences, University of Birmingham, Birmingham, UK

${ }^{2}$ School of Physical Therapy, Western University, London,

Ontario, Canada

${ }^{3}$ English Institute of Sport, The Manchester Institute of Health and Performance, Manchester, UK

Correspondence to

Dr Nicola R Heneghan;

n.heneghan@bham.ac.uk

\section{ABSTRACT}

Introduction Sports participation has many physical and mental health benefits for individuals with a disability, including improved functionality and reduced anxiety. Despite this, a large proportion of individuals with a disability are inactive. This review will be the first to synthesise the literature on the experiences and perceived health benefits of sport participation for children, adolescents, adults, elite athletes and veterans with a disability. Investigation of these phenomena will enable an understanding of the positive aspects and benefits of sport participation specific to each population, which may help to improve participation rates and ultimately improve health through promotion of these benefits.

Methods A protocol for systematic review is reported in line with Preferred Reporting Items for Systematic Reviews and Meta-Analysis-P. The phenomena of interest are the experiences and perceived health benefits of individuals with a disability participating in sport. There will be no age limit on participants and all study designs, besides reviews, will be included. Studies in languages other than English will be excluded. Two independent reviewers will conduct the searches, study selection, data collection and quality assessment independently. The online databases MEDLINE, EMBASE, PsychINF0, CINAHL Plus, Web of Science and SportDiscus will be electronically searched from database inception to February 2020. Grey literature will be searched and several sport-related journals will be hand-searched. The Quality Assessment Tool for Studies with Diverse Designs will be used for quality assessment of included studies. Thematic synthesis will be used to analyse the qualitative studies, narrative synthesis will be used to analyse the quantitative studies and the perceived health benefits will be analysed using content analysis. The strength of the overall body of evidence will be assessed and reported using GRADE-CERQual (Grading of Recommendations, Assessment, Development and Evaluation-Confidence in the Evidence from Reviews of Qualitative research) for qualitative studies and GRADE for quantitative studies. These approaches will be applied to mixed-methods studies, respectively, where necessary. Ethics and dissemination This systematic review raises no ethical issues. Results will be published in a peer reviewed journal and disseminated to key stakeholders to inform practice.

PROSPERO registration number CRD42020169224.
Strengths and limitations of this study

- This is the first systematic review to synthesise evidence on the experiences and perceived health benefits of individuals with a disability participating in sport.

- The research team includes researchers and practitioners with methodological and subject-specific expertise.

- Only articles written in English will be included in the analysis.

\section{INTRODUCTION}

Sport provides individuals with a disability with the opportunity to experience the many physical and mental health benefits associated with being physically active. ${ }^{1}$ These benefits include improved functionality, endurance and muscle tone, increased socialisation opportunities and a reduction in anxiety and depression across a range of disabilities and age groups. ${ }^{2-4}$ Despite the positive factors associated with sport participation, over $40 \%$ of adults with a disability are inactive in the UK, with similar figures reported in the USA $(44.3 \%) .^{5-7}$ Furthermore, individuals with a disability also have higher rates of chronic disease $\longrightarrow 40 \%$ of Americans with a disability develop heart disease, cancer, diabetes or have experienced a stroke compared with $<14 \%$ of those without a disability. ${ }^{6}$

The awareness of and participation in sport for individuals with a disability has grown in recent years as a result of the 'Paralympic Movement', which has been responsible for an increase in sporting opportunities, inclusion of individuals with a disability in sport and raising the profile of elite disability sport. ${ }^{7-9}$ This review will focus solely on sport participation, which will be defined as an activity involving physical exertion with or without a game or competition element, 
where skills and physical endurance are either required or to be improved. ${ }^{10}$

\section{Adults}

Over the past 3 years, the activity levels of adults with a disability have increased. ${ }^{11}$ Those completing $\geq 150 \mathrm{~min} /$ week have increased from $43.6 \%$ to $47.3 \%$, and those completing $<30 \mathrm{~min} /$ week have decreased from $42.4 \%$ to $39.8 \% .^{11}$ Similarly, in the USA, approximately $30 \%$ of adults with a disability have been found to regularly participate in sports or physical activity. ${ }^{12}$ Despite these positive trends in activity levels, surprisingly, the proportion of adults with an active sports club membership has decreased from $29.4 \%$ in $2017-2018$ to $21.4 \%$ in 2018-2019. ${ }^{11}$

\section{Children}

Children with a disability are more likely to be less active than their non-disabled peers, with one-third taking part in less than 30 min of physical activity per day ${ }^{13} 14$ $\left(\right.$ Sport England $^{5}$; Activity Alliance ${ }^{14}$ ). Additionally, several studies in a range of countries have reported low physical activity levels and high sedentary levels in children with a disability, suggesting that more needs to be done to promote their participation in sporting activities to improve overall health. ${ }^{15-19}$ However, statistics published in the UK in 2019 have shown that the inactivity levels of children with a disability aged 11-16 years have decreased compared with 12 months ago, from $38.1 \%$ to $34 \%$, suggesting an increase in participation. ${ }^{13}$

\section{Elite athletes}

At the elite level of sport, there has been a steady growth in participation at the Paralympic Games, increasing from around 3000 athletes and 83 countries at Barcelona in 1992 to over 4300 athletes and 160 countries in Rio $2016 .^{20}$ The funding for Paralympic sport has also grown, with UK Sport investing almost $£ 73$ million in the 4-year cycle leading up to the Rio Paralympic Games compared with just $£ 10$ million for the Sydney Paralympic Games cycle (2000). ${ }^{21}$ This greater awareness of and investment into elite disability sport has prompted research in this area, with studies exploring the beliefs, identities and selfperceptions of elite disability athletes. ${ }^{22-24}$ Despite this, there is still a relatively small body of research in elite sport, with limited research exploring the experiences of elite athletes with a disability.

\section{Veterans}

Sport participation has been shown to improve quality of life, increase confidence and provide a source of motivation for veterans with a disability. ${ }^{25} \mathrm{~A}$ systematic review has reported that sport and physical activity play a role in improving the well-being and rehabilitation of veterans after trauma and facilitating personal development. ${ }^{26}$ The authors of the systematic review proposed a potentially essential difference between 'sport' and 'physical activity' and the impact this may have on well-being, and suggested that future research should take this into consideration.
Furthermore, this review focused on the experiences of disability sport camps and competitions, with no review to date exploring the experiences and benefits of longer term sport participation in this population.

A review is required to synthesise the literature in this area as there is a limited understanding of the range of experiences and perceived health benefits of participation in these four populations. Understanding of these phenomena will enable the promotion of the health benefits and positive aspects of sport tailored to the specific populations. This may help to improve participation rates, ultimately improving the health and well-being of children, adolescents, adults and veterans. This review will also provide an insight into athletes' experiences at the elite level of sport, contributing to the small body of research, making recommendations for future research and enabling suggestions to improve performance.

\section{Objectives}

1. To explore the experiences and perceived health benefits of individuals with a disability participating in sport.

2. To explore the experiences of children and adolescents, adults, elite athletes and veterans with a disability participating in sport.

3. To examine the perceived health benefits of participating in sport for children and adolescents, adults, elite athletes and veterans with a disability.

\section{METHODS}

This systematic review protocol follows the Preferred Reporting Items of Systematic Review and Meta-Analysis Protocols (PRISMA-P) 2015 statement (see online supplemental file 1). ${ }^{27}$ This protocol and search has been designed involving subject-specific expertise in the form of an expert in the field of elite disability sport (PM) and methodological expertise in the form of extensive systematic review publications (AR, NRH and AS).

\section{Eligibility criteria}

Eligibility criteria are informed using the Sample, Phenomenon of Interest, Design, Evaluation and Research concept, which is designed for qualitative evidence synthesis. ${ }^{28}$ Studies will be eligible for inclusion in this review if they meet the following criteria:

Sample: studies which include individuals with a physical, visual or intellectual impairment who participate in sport, either competitively or recreationally. For studies with children and adolescents, the participants will be under 18 years of age; for studies with adults, the participants will be aged over 18 years; for studies with elite athletes, the participants will be of international standard or on the respective national team; and for studies with veterans, the participants will be ex-armed forces members. Studies which include individuals who are classed as disabled through old age or a medical condition in isolation (eg, diabetes) will be excluded. There is no age limit on participants. 
Phenomenon of Interest: the experiences of individuals with a disability participating in sport where experience includes aspects such as the meaning of sport, the support for participation and the barriers and facilitators to sport. The second phenomenon of interest is the perceived health benefits of sport, which include a participant's self-reported benefits and comments suggesting the benefits of sport. Perceived health benefits include physical health benefits such as increased muscle tone and weight management, and mental health benefits such as improved confidence and reduced anxiety. Studies investigating experiences and/or health benefits of a competition or sport programme less than 6 months in duration were excluded.

Designs: all types of study designs will be considered. Reviews will be excluded. Studies written in languages other than English will be excluded.

Evaluation: any reported experience by individuals with a disability in sport will be explored such as overall experiences, meaning, barriers and facilitators experienced in sport. The perceived health benefits of sport participation will be explored via studies, which have reported participant's perceived health benefits in form of a questionnaire or verbally reported benefits.

Research type: mixed-methods research.

\section{Information sources}

The databases MEDLINE (Ovid interface), EMBASE (Ovid interface), PyschINFO (Ovid interface), Web of Science (Clarivate Analytics interface), CINAHL Plus (EBSCO interface) and SportDiscus (EBSCO interface) will be searched from database inception to February 2020. Grey literature sources, including OpenGrey, will be searched. Hand searching of the following journals will be conducted to complement the search strategy: Qualitative Research in Sport, Exercise and Health, Psychology of Sport and Exercise, Disability and Rehabilitation, British Journal of Sports Medicine, European Journal of Sports Science and International Journal of Sports Science. The screening of the references of included studies will also take place. Active researchers who have published literature in this field will be contacted.

\section{Search strategy}

The search will be conducted independently by the lead author (BA, also the first reviewer) and a second reviewer. Initial scoping searches have refined the search terms for the databases, which will be kept broad to ensure a sensitive search strategy. Free text searches and subject heading searches will be carried out to ensure completeness of the search. The main body of the search strategy will be consistent across databases; however, specific search terms will be adjusted for each database to reflect syntax differences (see online supplemental file 2 for MEDLINE search strategy). ${ }^{29}$

\section{Study records}

Data management

The results of the literature search will be imported into EndNote V.X9, which will be used for data management and reference storage.$^{30}$ The reference, abstract and full text for all potentially eligible studies will be stored to allow effective screening. Any duplicates will be removed prior to the selection process.

\section{Selection process}

The lead author and a second reviewer will independently screen the titles and abstracts of studies at the same time to determine inclusion using the predetermined eligibility criteria. The eligibility criterion of eligible/not eligible/might be eligible will be used to assess the studies. Studies will be excluded if it is clear from the title and abstract that the content is not relevant to the objectives. When a study cannot be excluded based on the information provided in the title and abstract, it will be graded as 'might be eligible'. After title and abstract screening, full-text copies of the potentially relevant studies will be obtained and eligibility will be determined. Studies will also be removed if the information available is insufficient for assessment and synthesis, such as full-text copies not being available. These studies will not be included in the synthesis but may be referenced in the Discussion section. Consensus between the reviewers regarding study selection will be reached through a discussion, and in the case where an agreement is not reached, a third reviewer will be consulted. The kappa statistic will be used to test inter-rater reliability as it assesses the chance-corrected agreement between the two reviewers in assessing the eligibility of articles at the title/abstract stage and the fulltext screening stage. ${ }^{31}$ The study selection process will be carried out according to the PRISMA flow diagram and reported visually. ${ }^{32}$

\section{Data collection process}

The data will be extracted independently by the lead author and second reviewer from included studies using the standardised qualitative data extraction tool from the Joanna Briggs Institute (see online supplemental file 3) ${ }^{33}$ Piloting on five studies ahead of the main study will ensure completeness and suitability of the form. The form will be revised if necessary to include a section for study design, allowing the recording of whether the study is qualitative, quantitative or mixed methods in design. In the event of a disagreement between the two reviewers in data extracted, a third reviewer will be consulted.

\section{Data items}

The data extracted from the included studies will be presented in a table and the data items will include: participant information, data collection methods, data analysis methods and phenomenon of interest.

\section{Outcomes and prioritisation}

The experiences and perceived health benefits of children and adolescents, adults, elite athletes and veterans with a disability participating in sport constitute the phenomena of interest. All experiences reported by these individuals, including experiences of the benefits, 
barriers and facilitators to sports participation, will be explored provided that there is sufficient evidence.

\section{Quality assessment}

Initial scoping searches have suggested that studies with a range of designs will be eligible for inclusion in this systematic review. Therefore, the Quality Assessment Tool for Studies with Diverse Designs (QATSDD) will be used to create a quality rating score for all included studies (see online supplemental file 4). ${ }^{34}$ This tool is suitable for quality assessment because it allows the quality assessment of qualitative, quantitative and mixed-methods designs. ${ }^{34}$ The QATSDD allows the appraisal of qualitative research, which is vital for the qualitative research to contribute appropriately to the systematic review findings. ${ }^{35}$ Additionally, good validity, inter-rater reliability and test-retest reliability have been established with this tool and it allows an in-depth understanding of the included review papers. ${ }^{346}$ A summary of the quality score and converted percentage score for each study will be reported in a table. The lead author and second reviewer will independently carry out the quality assessment and if there is a lack of consensus between the two after a discussion, the third reviewer will be consulted. If additional information is required from authors, such as an interview topic guide, the authors will be contacted for this information to facilitate quality assessment.

\section{Data synthesis}

Studies will be categorised into one of the four population categories for analysis based on the participants. For mixed populations, if the ages of participants can be aligned with specific quotations or results, then the findings will be analysed in the respective population. The initial scoping searches demonstrated to the authors that both qualitative and quantitative studies would likely be included in the systematic review. Due to the potential heterogeneity in study designs, appropriate analysis methods will be required specific to the design. If mixedmethods studies are included, they will be analysed qualitatively and/or quantitatively according to the relevance of each phase to the review objectives.

Thematic synthesis is an appropriate method for the synthesis of qualitative evidence and is based on thematic analysis, which is used for the analysis of primary research. ${ }^{37} 38$ Therefore, included qualitative studies will be analysed following the stages suggested by Thomas and Harden ${ }^{37}$ for qualitative evidence synthesis in systematic reviews. The lead author (BA) will undertake line-byline coding of the text of included studies according to the content and meaning. ${ }^{37}$ Translation will be employed, which is the process of identifying concepts and ideas in one study and recognising them in another. ${ }^{39} \mathrm{~A}$ bank of codes will be created and maintained, which will then be grouped into descriptive themes based on connections between codes. ${ }^{37}$ The final stage will involve generating analytical themes through discussing findings with the research team and generating concepts which answer the review questions. ${ }^{37} 39$

A narrative synthesis will be conducted to analyse the quantitative studies. ${ }^{40}$ This will involve a preliminary synthesis of the results of included studies and an exploration of the relationships within and between studies by comparing the results and generating common themes. ${ }^{40}$ An integration matrix will be used to juxtapose the qualitative and quantitative data to determine agreement or disagreement within identified themes. ${ }^{41-43}$

The perceived health benefits of sport participation will be extracted either from questionnaires or verbally reported interview responses. The benefits will be analysed through content analysis, which involves coding and categorising data to determine the frequency and patterns of the health benefits across the different populations. ${ }^{44}$ The lead author will immerse herself in the data and focus on the manifest content of the data. ${ }^{44}$ This will involve analysing exactly what is said in the text and developing categories, which will be 'physical health benefits' and 'mental health benefits'. ${ }^{45}$ The thematic synthesis, narrative synthesis and content analysis will be conducted by the lead author and checked by two other authors with experience in these fields.

\section{Confidence in cumulative evidence}

To assess the overall quality and strength of evidence two different approaches will be utilised. The GRADECERQual (Grading of Recommendations, Assessment, Development and Evaluation-Confidence in the Evidence from Reviews of Qualitative research) will be used to assess how much confidence to place in the findings from the qualitative studies. ${ }^{46}$ This approach helps provide a transparent, systematic framework to guide the confidence in qualitative synthesis findings and has the potential to increase the usability of the findings from this systematic review. ${ }^{46}$ To assess the confidence in the findings from quantitative studies, the GRADE will be used. ${ }^{47}$ GRADE is used to rate the body of evidence at the outcome level, and is appropriate for use in this systematic review as it has been widely adopted to grade the quality of evidence, to make recommendations and to present summaries of evidence. ${ }^{48} 49$ The lead author will assess the overall body of evidence, which will be rated as 'high', 'moderate', 'low' or 'very low' based on the GRADE certainty ratings. ${ }^{48}$ A high rating would conclude that further research is not likely to greatly impact on confidence of findings and a low rating would suggest an uncertainty of effect and the need for further research. ${ }^{46} 48$

\section{DISCUSSION}

This systematic review will be the first to synthesise the literature on the experiences and perceived health benefits of individuals with a disability participating in sport. It will explore the sport experiences and health benefits in different populations, including children and adolescents, adults, elite athletes and veterans with a disability. At 
the end of the review, we will have some insight into both the positive and negative aspects experienced by individuals with a disability when participating in sport. It will provide more information about the meaning of sport, and the barriers and facilitators faced by individuals with a disability. This systematic review will also provide insight into how the sporting experience can be improved for each population based on the experiences reported, with the potential to increase participation in sport through awareness of the barriers faced and the promotion of the positive aspects of sport participation. The findings from this review will provide a clear basis and direction to guide further research based on the areas which are determined to require more investigation following data synthesis. Due to the four populations which will be included in this review, the future research directions and recommendations for practice will be population specific. This will enable specific research groups to take the findings and move forward with future research. This protocol provides a detailed account of the rationale and methods to be used in the proposed systematic review to ensure full transparency of the process. This study raises no ethical issues and any potential biases in the review process will be reported in the discussion section of the final review paper. Any required amendments to this protocol will be reported in the final systematic review and on PROSPERO along with the date, description and rationale for amendment.

\section{Patient and public involvement}

This study and protocol have been informed through extensive contact with key stakeholders in the field in both a professional physiotherapy and clinical capacity, and in an athletic capacity through contact with athletes with a disability. Since no individual data is needed, individuals with a disability will not be involved in data collection or analysis. Key stakeholders may be contacted for their input to the synthesis and interpretation of findings to inform results.

\section{Implications}

It is anticipated that the findings from this systematic review will provide an insight into the experiences and health benefits of participating in sport for individuals with a disability. It will provide insight into the meaning of sport, the barriers faced, facilitators increasing participation, and the physical and mental health benefits. Due to the exploration of these phenomena in the different population groups, the findings will be population specific and relevant to specific research groups, personalising the research needed going forward. This review will identify gaps in the evidence and suggest future research, and the findings may underpin policy decision-making for the provision of sport for individuals with a disability.

Twitter Alison Rushton @abrushton, Andrew Soundy @Andy_Soundy and Nicola R Heneghan @HeneghanNicola

Contributors $B A$ is a research student at the University of Birmingham. ABR, AS and NRH are supervisors. PM and NRH are experts in the field of disability sport.
$B A, A B R, A S, P M$ and NRH contributed to the systematic review topic. BA drafted the protocol with guidance and feedback from ABR, AS and NRH. ABR, PM, AS and NRH reviewed the manuscript and commented on the protocol. All authors have approved and contributed to the final manuscript.

Funding The authors have not declared a specific grant for this research from any funding agency in the public, commercial or not-for-profit sectors.

Competing interests None declared.

Patient consent for publication Not required.

Provenance and peer review Not commissioned; externally peer reviewed.

Supplemental material This content has been supplied by the author(s). It has not been vetted by BMJ Publishing Group Limited (BMJ) and may not have been peer-reviewed. Any opinions or recommendations discussed are solely those of the author(s) and are not endorsed by BMJ. BMJ disclaims all liability and responsibility arising from any reliance placed on the content. Where the content includes any translated material, BMJ does not warrant the accuracy and reliability of the translations (including but not limited to local regulations, clinical guidelines, terminology, drug names and drug dosages), and is not responsible for any error and/or omissions arising from translation and adaptation or otherwise.

Open access This is an open access article distributed in accordance with the Creative Commons Attribution Non Commercial (CC BY-NC 4.0) license, which permits others to distribute, remix, adapt, build upon this work non-commercially, and license their derivative works on different terms, provided the original work is properly cited, appropriate credit is given, any changes made indicated, and the use is non-commercial. See: http://creativecommons.org/licenses/by-nc/4.0/.

\section{ORCID iDs}

Alison Rushton http://orcid.org/0000-0001-8114-7669

Andrew Soundy http://orcid.org/0000-0002-5118-5872

Nicola R Heneghan http://orcid.org/0000-0001-7599-3674

\section{REFERENCES}

1 Slater D, Meade MA. Participation in recreation and sports for persons with spinal cord injury: review and recommendations. NeuroRehabilitation 2004;19:121-9.

2 Shephard RJ. Benefits of sport and physical activity for the disabled: implications for the individual and for Society. Scand J Rehabil Med 1991;23:51-9.

3 Barfield JP, Malone LA. Perceived exercise benefits and barriers among power wheelchair soccer players. J Rehabil Res Dev 2013;50:231-8.

4 Johnson CC. The benefits of physical activity for youth with developmental disabilities: a systematic review. Am J Health Promot 2009;23:157-67.

5 Sport England. Active lives adult survey may 18/19 report, 2019. Available: https://www.sportengland.org/media/14239/active-livesadult-may-18-19-report.pdf

6 Carroll DD, Courtney-Long EA, Stevens AC, et al. Vital signs: disability and physical activity-United States, 2009-2012. MMWR Morb Mortal Wkly Rep 2014;63:407-13.

7 International Paralympic Committee. History of the paralympic movement, 2019. Available: https://www.paralympic.org/ipc/history

8 Blauwet C, Willick SE. The Paralympic movement: using sports to promote health, disability rights, and social integration for athletes with disabilities. Pm R 2012;4:851-6.

9 Thomas N, Disability SA. Sport and society: an introduction. Oxon: Routledge, 2008.

10 Kemper HG, Ooijendijk WT, Stiggelbout M. Consensus about the dutch standard for healthy excercise. Tijdschrift voor Gezondheidswetenschappen 2000;78:180-3.

11 Sport England. Trends (UK active lives survey), 2020. Available: https://activelives.sportengland.org/Result?queryld=34030\#

12 Krane D, Orkis K. Sports and employment among Americans with disabilities:, 2020. Available: https://www.moveunitedsport.org/wpcontent/uploads/2015/11/Sports-and-Employment-Among-PeopleWith-Disabilities-2.pdf

13 Sport England. Active lives children and young people survey academic year 2018/19, 2020. Available: https://sportenglandproduction-files.s3.eu-west-2.amazonaws.com/s3fs-public/2020-01/ active-lives-children-survey-academic-year-18-19.pdf?cVMsdnpB oqROViY61iUjpQY6WcRyhtGs

14 Activity alliance. Fears for the future generation as report shows disabled children miss out, 2020. Available: http://www. 
activityalliance.org.uk/news/5668-fears-for-future-generation-asreport-shows-disabled-children-miss-out

15 Sit CHP, McManus A, McKenzie TL, et al. Physical activity levels of children in special schools. Prev Med 2007:45:424-31.

16 Sit CHP, McKenzie TL, Cerin E, et al. Physical activity and sedentary time among children with disabilities at school. Med Sci Sports Exerc 2017;49:292-7.

17 Sit C, Li R, McKenzie TL, et al. Physical activity of children with physical disabilities: associations with environmental and behavioral variables at home and school. Int J Environ Res Public Health 2019;16:1394.

18 Pan C-Y, Liu C-W, Chung IC, et al. Physical activity levels of adolescents with and without intellectual disabilities during physical education and recess. Res Dev Disabil 2015;36C:579-86.

19 Einarsson Ingi Ór, Ólafsson Ágúst, Hinriksdóttir G, et al. Differences in physical activity among youth with and without intellectual disability. Med Sci Sports Exerc 2015;47:411-8.

20 International Paralympic Committee. Summer paralympic games overview, 2019. Available: https://www.paralympic.org/paralympicgames/summer-overview

21 UK Sport. Historical funding figures, 2019. Available: https://www. uksport.gov.uk/our-work/investing-in-sport/historical-funding-figures

22 Pack S, Kelly S, Arvinen-Barrow M. "I think I became a swimmer rather than just someone with a disability swimming up and down:" paralympic athletes perceptions of self and identity development. Disabil Rehabil 2017;39:2063-70.

23 Kasum G, Mladenović M. The self-perception of athletes with disability. Fizicka kultura 2017;71:43-54.

24 de Cruz NP, Spray CM, Smith B. Implicit beliefs of disability and elite sport: the para-athlete experience. Qual Res Sport Exerc Health 2019;11:69-91.

25 Sporner ML, Fitzgerald SG, Dicianno BE, et al. Psychosocial impact of participation in the National veterans wheelchair games and winter sports clinic. Disabil Rehabil 2009;31:410-8.

26 Caddick N, Smith B. The impact of sport and physical activity on the well-being of combat veterans: a systematic review. Psychol Sport Exerc 2014:15:9-18.

27 Moher D, Shamseer L, Clarke M, et al. Preferred reporting items for systematic review and meta-analysis protocols (PRISMA-P) 2015 statement. Syst Rev 2015;4:1.

28 Cooke A, Smith D, Booth A. Beyond PICO: the spider tool for qualitative evidence synthesis. Qual Health Res 2012;22:1435-43.

29 Bramer WM, de Jonge GB, Rethlefsen ML, et al. A systematic approach to searching: an efficient and complete method to develop literature searches. J Med Libr Assoc 2018;106:531-41.

30 Clarivate. Endnote X9, 2019. Available: https://endnote.com/

$31 \mathrm{McHugh}$ ML. Interrater reliability: the kappa statistic. Biochem Med 2012:22:276-82.

32 Moher Det al. Preferred reporting items for systematic reviews and meta-analyses: the PRISMA statement. Ann Intern Med 2009;151:264-9.
33 Lockwood C, Porritt K, Munn Z, et al. Joanna Briggs Institute qualitative data extraction tool, 2019. Available: https://wiki. joannabriggs.org/display/MANUAL/Appendix+2.3\%3A+JBI+ Qualitative+data+extraction+tool

34 Sirriyeh R, Lawton R, Gardner P, et al. Reviewing studies with diverse designs: the development and evaluation of a new tool. J Eval Clin Pract 2012;18:746-52.

35 Dixon-Woods M, Sutton A, Shaw R, et al. Appraising qualitative research for inclusion in systematic reviews: a quantitative and qualitative comparison of three methods. J Health Serv Res Policy 2007;12:42-7.

36 Fenton L, Lauckner H, Gilbert R. The QATSDD critical appraisal tool: comments and critiques. J Eval Clin Pract 2015;21:1125-8.

37 Thomas J, Harden A. Methods for the thematic synthesis of qualitative research in systematic reviews. BMC Med Res Methodol 2008;8:45.

38 Braun V, Clarke V. Using thematic analysis in psychology. Qual Res Psychol 2006;3:77-101.

39 Britten N, Campbell R, Pope C, et al. Using meta ethnography to synthesise qualitative research: a worked example. J Health Serv Res Policy 2002;7:209-15.

40 Popay J, Roberts H, Sowden A, et al. Guidance on the conduct of narrative synthesis in systematic reviews, 2020. Available: https://www.researchgate.net/profile/Mark_Rodgers4/publication/ 233866356_Guidance_on_the_conduct_of_narrative_synthesis in systematic reviews A product from the ESRC Methods Programme/links/02e7e5231e8f3a6183000000/Guidance-on-theconduct-of-narrative-synthesis-in-systematic-reviews-A-productfrom-the-ESRC-Methods-Programme.pdf

41 Thomas J, Harden A, Oakley A, et al. Integrating qualitative research with trials in systematic reviews. BMJ 2004;328:1010-2.

42 Sandelowski M, Voils Cl, Barroso J. Defining and designing mixed research synthesis studies. Res Sch 2006;13:29.

43 Caracelli VJ, Greene JC. Data analysis strategies for mixed-method evaluation designs. Educ Eval Policy Anal 1993;15:195-207.

44 Vaismoradi $\mathrm{M}$, Turunen $\mathrm{H}$, Bondas $\mathrm{T}$. Content analysis and thematic analysis: implications for conducting a qualitative descriptive study. Nurs Health Sci 2013;15:398-405.

45 Elo S, Kyngäs $\mathrm{H}$. The qualitative content analysis process. J Adv Nurs 2008;62:107-15

46 Lewin S, Booth A, Glenton C, et al. Applying GRADE-CERQual to qualitative evidence synthesis findings: introduction to the series. Implementation Sci 2018;13:1-10.

47 Guyatt G, Oxman AD, Akl EA, et al. Grade guidelines: 1. IntroductionGRADE evidence profiles and summary of findings tables. J Clin Epidemiol 2011;64:383-94.

48 Siemieniuk R, Guyatt G. What is grade? 2020. Available: https:// bestpractice.bmj.com/info/toolkit/learn-ebm/what-is-grade/

49 Guyatt GH, Oxman AD, Vist GE, et al. Grade: an emerging consensus on rating quality of evidence and strength of recommendations. $B M J$ 2008;336:924-6. 